\title{
Plasmatic villin 1 is a novel in vivo marker of proximal tubular cell injury during renal ischemia-reperfusion.
}

\author{
Jean-Paul Decuypere, $\mathrm{PhD}^{1 \dagger}$, Laurens J. Ceulemans, MD, $\mathrm{PhD}^{1}$, Tine Wylin $\mathrm{PhD}^{1}$, \\ Martinet Wim, $\mathrm{PhD}^{2}$, Diethard Monbaliu, $\mathrm{MD}, \mathrm{PhD}^{1}$, Jacques Pirenne, $\mathrm{MD}, \mathrm{PhD}^{1}$, \\ Ina Jochmans MD, $\mathrm{PhD}^{* 1}$
}

\begin{abstract}
Affiliation:
${ }^{1}$ Laboratory of Abdominal Transplantation, Department of Microbiology and Immunology, KU Leuven \& Department of Abdominal Transplant Surgery, University Hospitals Leuven, Leuven, Belgium

${ }^{2}$ Laboratory of Physiopharmacology, Department of Pharmaceutical Sciences, University of Antwerp, Antwerp, Belgium

${ }^{\dagger}$ Current affiliation: VIB Center for Brain \& Disease Research, Laboratory for Membrane Transport; KU Leuven, Department of Neurosciences, Leuven, Belgium
\end{abstract}

\section{*Correspondence:}

Ina Jochmans, MD, PhD

Abdominal Transplant Surgery

University Hospitals Leuven

Herestraat 49 box 7003

3000 Leuven Belgium

ina.jochmans@uzleuven.be 


\section{Authorship}

JPD designed the study, acquired, analyzed and interpreted the data, wrote the manuscript and takes responsibility for the integrity of the data and the accuracy of the data analysis.

LJC, TW, WM, DM, JP and IJ contributed to the design of the study, acquisition, analysis and interpretation of data, critically revised and approved the manuscript and take responsibility for the integrity of the data and the accuracy of the data analysis.

\section{Disclosure}

JP holds a named chair at the KU Leuven from the Institut Georges Lopez. JP, DM, and IJ hold a named chair at the KU Leuven from the "Centrale Afdeling voor Fractionering".

\section{Funding}

At the time of the presented research, JPD was a fellow of the European Renal Association European Dialysis and Transplant Association (ERA-EDTA). The work was supported by a Research Grant of the Research Foundation Flanders (FWO - KaN 1518914N). 


\section{Abbreviations}

AFP: alpha-fetoprotein

AKI: acute kidney injury

AST: aspartate transaminase

h-FABP: heart-type fatty acid-binding protein

IHC: Immunohistochemistry

KIM-1: Kidney Injury Molecule-1

MP: milk powder

Nec-1: necrostatin-1

NGAL: neutrophil gelatinase-associated lipocalin

PBS: phosphate-buffered saline

RIP: receptor-interacting protein

SDS-PAGE: sodium dodecyl sulfate - polyacrylamide gel electrophoresis 


\section{Abstract}

Background: Early detection of acute kidney injury (AKI), a common condition with a high mortality risk, can be facilitated by specific and reliable biomarkers. Villin 1, a protein typically found in the brush borders of proximal tubular cells, has been detected in urine of patients with AKI, but its possible release in plasma remains unexplored. Methods: We measured the presence of villin 1 by immunohistochemistry on kidney sections and by Western blotting in plasma samples from rats subjected to renal ischemia-reperfusion injury, pigs subjected to renal transplantation and liver transplantation patients that developed AKI. Moreover, rats were treated with necrostatin-1, an inhibitor of programmed necrosis (necroptosis), which occurs in renal tubular cells during AKI. Villin 1 levels were compared to other renal injury markers (creatinine, aspartate transaminase and heart-type fatty acid binding protein). Results: During AKI, plasmatic villin 1 levels corresponded with the severity of kidney injury and dysfunction. Its detection in plasma was associated with a redistribution in the kidney tissue. Unlike the levels of other markers, plasmatic villin 1 decreased already after a short $(3 \mathrm{~h})$ treatment with necrostatin1 during renal ischemia-reperfusion injury. The presence of plasmatic villin 1 was confirmed in patients who experienced AKI after liver transplantation. Conclusions: Villin 1 is released in plasma during AKI and shows potential as an early marker for proximal tubular injury/necrosis and warrants further investigation. 


\section{Introduction}

Currently available biomarkers for acute kidney injury (AKI) are hampered by the inability to distinguish between histological AKI (ie, intrinsic kidney damage) and functional AKI. ${ }^{1}$ Quantification of the extent of tubular injury or necrosis is not possible without an invasive tissue biopsy. Although several proteins are released into the blood or urine during kidney injury, ${ }^{1,2}$ it is unclear whether these are markers of necrosis or of possible reversible cellular injury. Detection of a plasma protein released only upon tubular damage would be very valuable in both clinical and experimental settings. Villin 1 is such a potential selective marker.

Villin 1 is part of the cytoskeleton of the microvilli of epithelial brush borders of enterocytes and the kidney's proximal tubular cells. Villin 1 has been detected in urine of patients with AKI; in 1 case following hemolytic uremic syndrome and in 2 cadaveric kidney transplant recipients. ${ }^{3}$ Additionally, villin 1 in the proximal tubular cells of rats appears to be redistributed during AKI from the brush borders towards the basolateral membrane. ${ }^{4}$ This redistribution positions villin 1 closer to the renal capillaries and suggests that it could be released in the blood as well. The detection of villin 1 in blood would be preferred, since collection of urine is not straightforward in cases of anuric or oliguric AKI. In addition, since villin 1 is a large $(92.5 \mathrm{kDa})$ cytoskeletal protein, its release in the blood could mark tubular necrosis rather than release through active exocytosis.

We therefore investigated whether villin 1 is detectable in plasma during AKI, reflects severity of kidney injury, and is associated with tubular necrosis. 


\section{Materials and Methods}

The animal care and experimental protocols were in accordance with the European guidelines and approved by the ethical committee of KU Leuven. The use of human samples was approved by the ethical committee of the UZ Leuven.

\section{Acute kidney injury in rats}

Female Sprague-Dawley rats of 200-250g were anesthetized with an intraperitoneal injection consisting of $7.5 \mathrm{mg} / \mathrm{kg}$ ketamin (Anesketin, Eurovet) and 2.5mg/kg xylazin (Xyl-M 2\%, Van Miert \& Dams Chemie (VMD)). After a midline laparotomy, both renal pedicles were dissected and clamped with microaneurysm clamps to induce ischemia. Three groups were considered based on the duration of ischemia: 0 min (sham-operated; sham), 45 min (I45) and 60 min (I60). These groups were further divided into subgroups with various reperfusion (R) times: R0h, R1h, R3h, R6h, R24h, R7d (N=6 per group). Analgesics were administered daily (Vetergesic $0.1 \mathrm{mg} / \mathrm{kg} \mathrm{bw}$ ). For sacrifice, rats were administered Nembutal (60 mg/kg bw) intraperitoneally and plasma was collected after the allotted reperfusion time.

\section{Kidney autotransplantation in pigs}

Procedures have previously been described. ${ }^{2,5}$ Briefly, female Landrace pigs (24-51 kg, ca. 12 weeks) underwent a left ureteronephrectomy 1 day before transplantation $(-24 \mathrm{~h})$. The procured kidney graft was subjected to 0,30 or 60 min warm ischemia (minimally, moderately and severely injured grafts, respectively) by clamping the renal artery and vein and subsequently cold stored in University of Wisconsin preservation solution ( $\mathrm{N}=6$ per group, $\mathrm{N}=7$ for severely injured grafts). An autotransplantation was performed 18 hours later, following contralateral 
nephrectomy. Plasma samples were collected at different time points postreperfusion: 15 min, 1 h, $3 \mathrm{~h}, 6 \mathrm{~h}$ and every following day (24 - $192 \mathrm{~h})$.

\section{Patients}

Patient samples were selected from a prospective cohort study investigating the occurrence of AKI after liver transplantation, described previously. ${ }^{6}$ Plasma samples taken at $2 \mathrm{~h}$, $6 \mathrm{~h}$, and $12 \mathrm{~h}$ after liver reperfusion were analyzed. Patients were followed until 1 year after liver transplantation. The occurrence of AKI was assessed by the RIFLE criteria at each time point during follow-up.

\section{Kidney function and injury markers}

Plasmatic heart fatty acid binding protein (h-FABP) was detected with a rat h-FABP ELISA (both from Hycult Biotech), while aspartate aminotransferase (AST) and creatinine were detected with IFCC colorimetric assay and the kinetic Jaffé method, respectively (both from Roche Diagnostics) in the central lab of the UZ Leuven. In human samples, urinary kidney injuy molecule-1 (KIM-1) and neutrophil-gelatinase associated lipocalin (NGAL) were measured by ELISA per the manufacturer's instructions (KIM-1 (human) ELISA kit, dilution 1/500, detection limit 1.279 pg/ml, Enzo Life Sciences, Lause, Switzerlandand - NGAL (human) ELISA KIT;

dilution 1/500, detection limit $4 \mathrm{pg} / \mathrm{mL}$; Bioporto, Gentofte, Denmark). Plasma h-FABP was measured by ELISA per the manufacturer's instructions (dilution 1/10; ELISA HK402, detection limit 102 pg/mL, Hycult Biotech, Uden, the Netherlands). 


\section{Western blotting}

Protein concentration of plasma samples was determined through use of Bradford protein assay. Fifty $\mu \mathrm{g}$ of protein was then prepared with Laemmli buffer containing $\beta$ mercaptoethanol, heated at $95^{\circ} \mathrm{C}$ for $3 \mathrm{~min}$ and loaded on Any $\mathrm{kD}$ Mini-Protean TGX Precast Gel (Bio-Rad Laboratories). SDS-PAGE was performed with a constant voltage of $150 \mathrm{~V}$ for ca. $45 \mathrm{~min}$. Next, proteins were blotted on polyvinylidene difluoride (PVDF) membranes using the semi-dry Trans-Blot Turbo Transfer system (Bio-Rad Laboratories). After transfer, the membranes were stained with Ponceau S solution to confirm equal loading in all lanes. Membranes were blocked for $1 \mathrm{~h}$ at room temperature with PBS-Tween (0.1\%) containing 5\% milk powder (MP) followed by incubation with the primary antibody against villin 1 (Cell Signaling Technologies, Danvers, MA, USA) or alpha-fetoprotein (AFP, Novus Biologicals, Littleton, CO, USA) diluted in PBS-T and $2 \% \mathrm{MP}$ overnight at $4^{\circ} \mathrm{C}$. Next day, the membranes were washed 3 times with PBS-T for 5 min and then incubated with the secondary horseradish peroxidase (HRP)-coupled antibody for 45 min with PBS-T $+2 \%$ MP. Blots were washed 3 times for 5 min with PBS-T. Immunoreactive bands were visualized through enhanced chemoluminescence (Pierce ECL Western Blotting Substrate), followed by detection and band intensity quantification using the Chemidoc MP technology and associated Imagelab software (Bio-Rad Laboratories). Band intensity was quantified and normalized to Sham/background levels.

\section{Villin 1 immunohistochemistry}

Right kidneys were removed after euthanasia of the rats and were fixed in formalin at $4^{\circ} \mathrm{C}$, embedded in paraffin using the Thermo Scientific Microm STP 120 Spin Tissue Processor. Sections of $\sim 4 \mu \mathrm{m}$ thickness were cut with a microtome and transferred to a microscope slide. 
After 60 min incubation in Target Retrieval Solution, High $\mathrm{pH}$ (Dako, Agilent), at $98^{\circ} \mathrm{C}$ in a PT Link machine (Dako), the sections were washed twice with Envision FLEX Washing buffer, followed by incubation with peroxidase blocking reagent for $5 \mathrm{~min}$. After 2 new wash steps, the sections were incubated for $30 \mathrm{~min}$ in the primary antibody solution (1:100), followed by $30 \mathrm{~min}$ in the secondary antibody solution (EnVision FLEX/HRP). Finally, the sections were incubated for $30 \mathrm{~min}$ in diaminobenzidine solution. The counterstaining (nuclei) is done automatically by the Leica ST5010 Autostainer XL. Slides were observed with an Olympus BX61 microscope at 20x magnification and pictures were taken with the Olympus Stream Essentials 1.9 software.

\section{TUNEL staining}

For the detection of oligonucleosomal DNA cleavage via terminal deoxynucleotidyl transferase end labelling (TUNEL), tissue sections were deparaffinized in toluene ( 2 × $5 \mathrm{~min}$ ), rehydrated in distilled water ( $5 \mathrm{~min}$ ) and pretreated with $3 \%$ citric acid (60 min) to remove tissue calcification. Endogenous peroxidase was quenched by incubating sections for $15 \mathrm{~min}$ in $0.9 \%$ hydrogen peroxide. Thereafter, TUNEL was performed using an ApopTag Plus Peroxidase In Situ Apoptosis Detection Kit (Millipore) according to the instructions of the manufacturer. Slides were observed as described above. Quantification of the TUNEL staining was done with the 'color threshold' function of ImageJ, using color space RGB and the same parameters for each analyzed picture.

\section{Statistical analysis}

Normality of the data and differences in variances were first assessed with the Kolmogorov-Smirnov test and Levene's test, respectively. Unpaired t tests, 1-Way ANOVA (in 
case of normal distribution) or Kruskal-Wallis (when not normally distributed) tests were performed where appropriate (analysis performed with GraphPad Prism 5).

\section{Results}

\section{Villin 1 is redistributed and released in plasma upon AKI}

AKI was induced in rats by bilateral clamping of renal arteries and veins for $45 \mathrm{~min}$ followed by reperfusion for 1 h, 3 h, 6 h, 24 h, or 7 days. Sham-operated rats underwent the same procedure but without clamping of the renal pedicle. This ischemia-reperfusion injury resulted in AKI as shown by significantly increased creatinine concentrations compared to sham after $1 \mathrm{~h}, 3$ $\mathrm{h}$ and $6 \mathrm{~h}$ of reperfusion (Figure 1D). AKI was reversible with creatinine values comparable to sham at $24 \mathrm{~h}$ and 7 days postreperfusion (Figure 1D). Plasma samples were analyzed with a potent villin 1-specific antibody and an antibody against AFP as housekeeping protein. While villin 1 was undetectable in Sham, it emerged in plasma at $1 \mathrm{~h}$ and $3 \mathrm{~h}$ after onset of AKI (Figure 1A), corresponding with increased creatinine and the release of other kidney injury markers hFABP and AST (Figure 1B-D). Immunohistochemistry (IHC) staining of villin 1 confirmed the brush border-restricted localization of villin 1 in Sham. However, after AKI this restricted staining was less clear, as villin 1 appeared more evenly distributed over the proximal tubuli, including cytoplasmic staining (Figure 1E).

Additionally, we also observed the appearance of plasmatic villin 1 when moderately or severely injured kidney grafts were transplanted in a porcine kidney autotransplantation model (see Figure S1, SDC, http://links.lww.com/TP/B465). ${ }^{2}$ In this model, a minimally injured kidney graft experiences ischemia-reperfusion injury after $18 \mathrm{~h}$ cold preservation which results in 
histological AKI after transplantation but only with a limited functional effect as grafts continued to produce urine and creatinine increase was only mild and transient (see Figure S1D, SDC, http://links.lww.com/TP/B465 minimally injured grafts). Plasma h-FABP and AST were detected in moderate concentrations and villin 1 remained undetected (see Figure S1A-C, SDC, http://links.lww.com/TP/B465 minimally injured grafts). When the renal vessels were clamped during donor surgery, eliciting warm ischemia and therefore additional injury, graft function decreased (see Figure S1D, SDC, http://links.lww.com/TP/B465 moderately -30 min warm ischemia- and severely -60 min warm ischemia- injured grafts) while a comparable grade of injury was seen on biopsy. ${ }^{2}$ In these cases of extended kidney injury, villin 1 could be detected in the plasma of these animals early after reperfusion in addition to AST and h-FABP (see Figure S1A-C, SDC, http://links.lww.com/TP/B465 moderately and severely injured grafts). For WB detection of the protein, shorter exposure time was needed to detect plasmatic villin 1 in samples from animals subjected to longer warm ischemia durations, suggesting that the levels of plasmatic villin 1 reflect the severity of AKI.

\section{Plasmatic villin 1 levels reflect the severity of renal injury}

To analyze whether the amount of villin 1 indeed reflects the severity of injury, we measured plasmatic villin 1 in a rat model with increased ischemic stress (45 vs 60 min of clamping). Like h-FABP and AST, significantly more villin 1 was detected in the plasma after $60 \mathrm{~min}$ of ischemia followed by $3 \mathrm{~h}$ of reperfusion compared to $45 \mathrm{~min}$ of ischemia and $3 \mathrm{~h}$ of reperfusion (Figure 2). 


\section{Release of villin 1 in the plasma is associated with necrosis}

Proximal tubular cells undergo programmed necrosis mediated by RIP kinases (termed necroptosis) during ischemic AKI. ${ }^{7}$ Since we hypothesized that the plasmatic release of villin 1 is the result of necrotic destruction of proximal tubular cells, we inhibited necroptosis by injecting $1.7 \mathrm{mg} / \mathrm{kg}$ of the necroptosis inhibitor Necrostatin-1 (Nec-1) intraperitoneally $1 \mathrm{~h}$ before 45 min renal ischemia and collected plasma $3 \mathrm{~h}$ after reperfusion. Reperfusion time was deliberately kept short to distinguish between direct and indirect (eg, attenuation of inflammation) effects of necrosis inhibition. Nec-1 indeed counteracted renal injury, as the positive TUNEL staining observed after $3 \mathrm{~h}$ of reperfusion was clearly decreased after Nec-1 treatment (Figure 3E). It should be noted that besides the expected TUNEL staining of the nuclei (white arrows in Figure 3E), we also consistently observed staining of the tubular lumenl. Interestingly, Nec-1 administration resulted in significantly less plasmatic villin 1 compared to vehicle (Figure 3A). In contrast, plasmatic h-FABP, AST and creatinine were not significantly altered by Nec-1 (Figure 3B-D) after this short reperfusion period, suggesting that among the detected renal injury markers, the amount of plasmatic villin 1 is most dependent on the extent of proximal tubular cell necrosis.

\section{Plasmatic villin 1 release in patients suffering from AKI after liver transplantation}

As a proof-of-principle, we sought to detect villin 1 in the plasma of patients that experienced AKI. For this purpose, we analyzed plasma samples from 2 liver transplantation patients that developed AKI shortly after liver reperfusion (patient 2, AKI at 24h; patient 3 at 12h) and compared it with a liver transplantation patient that did not develop AKI (patient 1). Graft and patient survival for all 3 patients at 1 year after transplantation was $100 \%$. Baseline 
donor and recipient characteristics can be found in Table 1. Plasmatic release of villin 1 could be detected in the 2 AKI patients as early as $2 \mathrm{~h}$ after reperfusion but not in the patient that did not develop AKI (Figure 4). It is important to note that the AFP levels decreased in the AKI patients (for reasons currently unknown), but AFP detection remained similar in the non-AKI patient. Interestingly, the levels of the other injury markers measured (plasma h-FABP, urine NGAL and urine KIM-1 - Table 1) did not unequivocally reflect injury in these 2 AKI patients, suggesting villin 1 could be an interesting additional kidney injury marker.

\section{Discussion}

Taken together, this data suggests that upon kidney injury, the proximal-tubule-specific protein villin 1 is redistributed in the cell and subsequently released in plasma, from where it can be easily collected and measured - even in the absence of urine production. Since the levels of villin 1 seem to increase in line with the severity of kidney injury and because it can be detected in patients as well, our data suggest that it has potential as a kidney injury marker that could be clinically exploited. Furthermore, as villin 1 was the only marker significantly decreased after short treatment with a necroptosis inhibitor, it might prove to be a specific marker for tubular necrosis, while other markers might be released upon sublethal cellular injury.

\section{Distribution of villin 1 during AKI}

Interestingly, it seems that 2 pools of circulating villin 1 can be detected after AKI: 1 in urine $^{3}$ and another in plasma (Figure 1). This distinction likely represents the intracellular redistribution of villin 1 observed during AKI: 1 fraction of villin 1 is transported towards the

basolateral membrane (Figure $1 \mathrm{E})^{4}$ and released in the capillaries, while another fraction remains 
at the apical membrane and will be released in the urine. Whether the urinary release of villin 1 is also of a likely necrotic nature needs investigation. Moreover, it is noteworthy that plasmatic villin 1 was always detected as a single ca. $92.5 \mathrm{kDa}$ protein, unlike in the urine where degradation fragments (of 70, 45 and $22 \mathrm{kDa}$ ) were observed. ${ }^{3}$ These degradation fragments are possibly the result of meprins, metalloproteinases abundantly expressed in the brush border membranes, which have been shown to cleave villin 1 during renal ischemia-reperfusion injury. ${ }^{8}$ As meprins are not present on the basolateral membrane, villin 1 in the plasma is thus only present as the full-length protein.

\section{Plasmatic villin 1 release is associated with cell death}

Proximal tubular cells are suggested to be heavily subjected to apoptosis, necrosis and programmed necrosis (necroptosis) during AKI. This is reflected in the 'atypical' positive TUNEL staining in rat kidney $3 \mathrm{~h}$ postreperfusion (Figure 3E): staining of the lumen of tubuli was consistently observed together with the typical nuclear staining, a feature which was

previously also described. ${ }^{9}$ As it was strongly attenuated by Nec-1, this atypical staining seems to be a consequence of necroptosis. Interestingly, villin 1 was the only biomarker affected shortly (only $3 \mathrm{~h}$ ) after reperfusion upon treatment with Nec-1 (Figure 3). As such, villin 1 is most dependent on necroptosis and is strongly associated with tubular cell death.

\section{Villin 1 as a potential clinical biomarker of kidney injury}

Several biomarkers are currently assessed in the clinic to estimate the extent of kidney injury. However, measurement of urinary biomarkers is often not feasible (due to oligo- or anuria) and the combination of multiple biomarkers is required to get a proper estimate, as each 
biomarker tends to vary from patient to patient. This is also reflected in Table 1, where none of the biomarkers measured show consistently higher levels in the 2 AKI patients compared to the non-AKI patient. In this respect, villin 1 has the potential of being a reliable early biomarker reflecting kidney proximal tubular cell death and therefore kidney injury. However, 2 limitations currently shadow the clinical use of villin 1: a) its detection is currently only semi-quantitative by WB and requires a more quantitative approach (eg, ELISA) and; b) its clearance is very fast and this mechanism should be better understood and potentially inhibited. Although speculative, clearance of villin 1 might not occur via the liver, as the liver transplantation patients that developed AKI also developed early allograft dysfunction but still showed fast clearance of villin $16 \mathrm{~h}$ after hepatic reperfusion.

The exact value of villin 1, its possible role in AKI and how it compares with the other kidney function/injury markers, such as kidney-injury molecule 1 or neutrophil gelatinase associated lipocalin, warrants further investigation. Currently, we were only able to detect villin 1 by Western blotting, which is time-consuming and semi-quantitative. The development of a quantitative high-throughput method would certainly be useful to expand on plasmatic or urinary villin 1's potency as an in vivo necrotic kidney injury marker. 


\section{References}

1. Gentile G, Remuzzi G. Novel Biomarkers for Renal Diseases? None for the Moment (but One). J Biomol Screen. 2016; 21(7):655-70.

2. Jochmans I, Lerut E, van Pelt J, Monbaliu D, Pirenne J. Circulating AST, H-FABP, and NGAL are early and accurate biomarkers of graft injury and dysfunction in a preclinical model of kidney transplantation. Ann Surg. 2011; 254(5):784-91.

3. Zimmerhackl LB, Leuk B, Hoschutzky H. The cytoskeletal protein villin as a parameter for early detection of tubular damage in the human kidney. J Chromatogr. 1991; 587(1):81-4.

4. Brown D, Lee R, Bonventre JV. Redistribution of villin to proximal tubule basolateral membranes after ischemia and reperfusion. Am J Physiol. 1997; 273(6):F1003-12.

5. Jochmans I, Lerut E, Heedfeld V, Wylin T, Pirenne J, Monbaliu D. Reproducible model for kidney autotransplantation in pigs. Transplant Proc. 2009; 41(8):3417-21.

6. Jochmans I, Meurisse N, Neyrinck A, Verhaegen M, Monbaliu D, Pirenne J. Hepatic ischemia/reperfusion injury associates with acute kidney injury in liver transplantation: prospective cohort study. Liver Transpl. 2017; 23(5):634-44.

7. Linkermann A, Brasen JH, Himmerkus N, et al. Rip1 (receptor-interacting protein kinase 1) mediates necroptosis and contributes to renal ischemia/reperfusion injury. Kidney Int. 2012; 81(8):751-61.

8. Ongeri EM, Anyanwu O, Reeves WB, Bond JS. Villin and actin in the mouse kidney brush-border membrane bind to and are degraded by meprins, an interaction that contributes to injury in ischemia-reperfusion. Am J Physiol Renal Physiol. 2011; 301(4):F871-82. 
9. Mulay SR, Thomasova D, Ryu M, Anders HJ. MDM2 (murine double minute-2) links inflammation and tubular cell healing during acute kidney injury in mice. Kidney Int. 2012; 81(12):1199-211. 


\section{Figure Legends}

Figure 1: Villin 1 is released in plasma during ischemic acute kidney injury in rats. Rats were subjected to Sham operation (Sham) or 45 min of renal ischemia (I45) followed by the annotated time of reperfusion (R0h, R1h, R3h, R6h, R24h, R7d). Plasma was collected and tested for (A) villin 1 and alpha-fetoprotein (AFP) by Western blotting; (B) aspartate transaminase, AST; (C) heart-type fatty acid-binding protein, h-FABP and; (D) creatinine. (E) Antivillin 1 immunohistochemistry staining of kidney sections of rats subjected to Sham operation, $1 \mathrm{~h}$ or $7 \mathrm{~d}$ or reperfusion (R1h, R7d). Representative pictures are shown from 2 independently collected rat kidney sections. Scale bar represents $150 \mu \mathrm{m}$. Insets (in red) are magnified below. White arrows indicate cytoplasmic villin 1 staining. *,**, *** p values $<0.05,<0.01,<0.001$, respectively, as analyzed by 1-Way ANOVA (in case of normal distribution) or Kruskal-Wallis (when not normally distributed) test for comparison of multiple groups ( $N=6$ per group).

Figure 2: Plasmatic villin 1 levels are dependent on the extent of renal injury. Rats were subjected to $3 \mathrm{~h}$ of reperfusion following 45 (I45) or 60 (I60) min of ischemia (I45R3h vs I60R3h). Plasma was collected and tested for (A) villin 1 and alpha-fetoprotein (AFP) by Western blotting; (B) aspartate transaminase, AST; (C) heart-type fatty acid-binding protein, hFABP and; (D) creatinine. *, ** p values $<0.05,<0.01$, respectively, as analyzed by an unpaired $\mathrm{t}$ test (when normally distributed) or Mann-Whitney test (when not normally ditributed) ( $N=6$ per group). 
Figure 3: Plasmatic villin 1 levels during acute kidney injury are decreased by treatment with necroptosis inhibitor Necrostatin-1. Rats were subjected to $45 \mathrm{~min}$ of ischemia followed by $3 \mathrm{~h}$ of reperfusion (I45R3h) after administration of Vehicle $(+\mathrm{Veh})$ or $1.7 \mathrm{mg} / \mathrm{kg}$ Necrostatin-1 (Nec1). Plasma was collected and tested for (A) villin 1 and alpha-fetoprotein (AFP) by Western blotting; (B) aspartate transaminase, AST; (C) heart-type fatty acid-binding protein, h-FABP and; (D) creatinine. (E) TUNEL staining of kidney sections of Vehicle- or Nec-1-treated rats and quantification of the area of positive staining (percentage). Scale bar represents $150 \mu \mathrm{m}$. White arrows indicate TUNEL-positive nuclei. $* \mathrm{p}$ values $<0.05$, as analyzed by unpaired $\mathrm{t}$ test (all normally distributed) ( $N=6$ per group).

Figure 4: Plasmatic villin 1 release in liver transplantation patients that experienced acute kidney injury (AKI). Plasma from liver transplantation patients that did (AKI) or did not (nonAKI) develop AKI was collected before incision (-; baseline) and 2, 6 and $12 \mathrm{~h}$ after reperfusion and analyzed for villin 1 and alpha-fetoprotein (AFP) by Western blotting. As AFP was not stable, Ponceau S staining of proteins was performed to confirm equal loading. The protein marker is indicated by ' $\mathrm{M}$ '. 
Figure 1
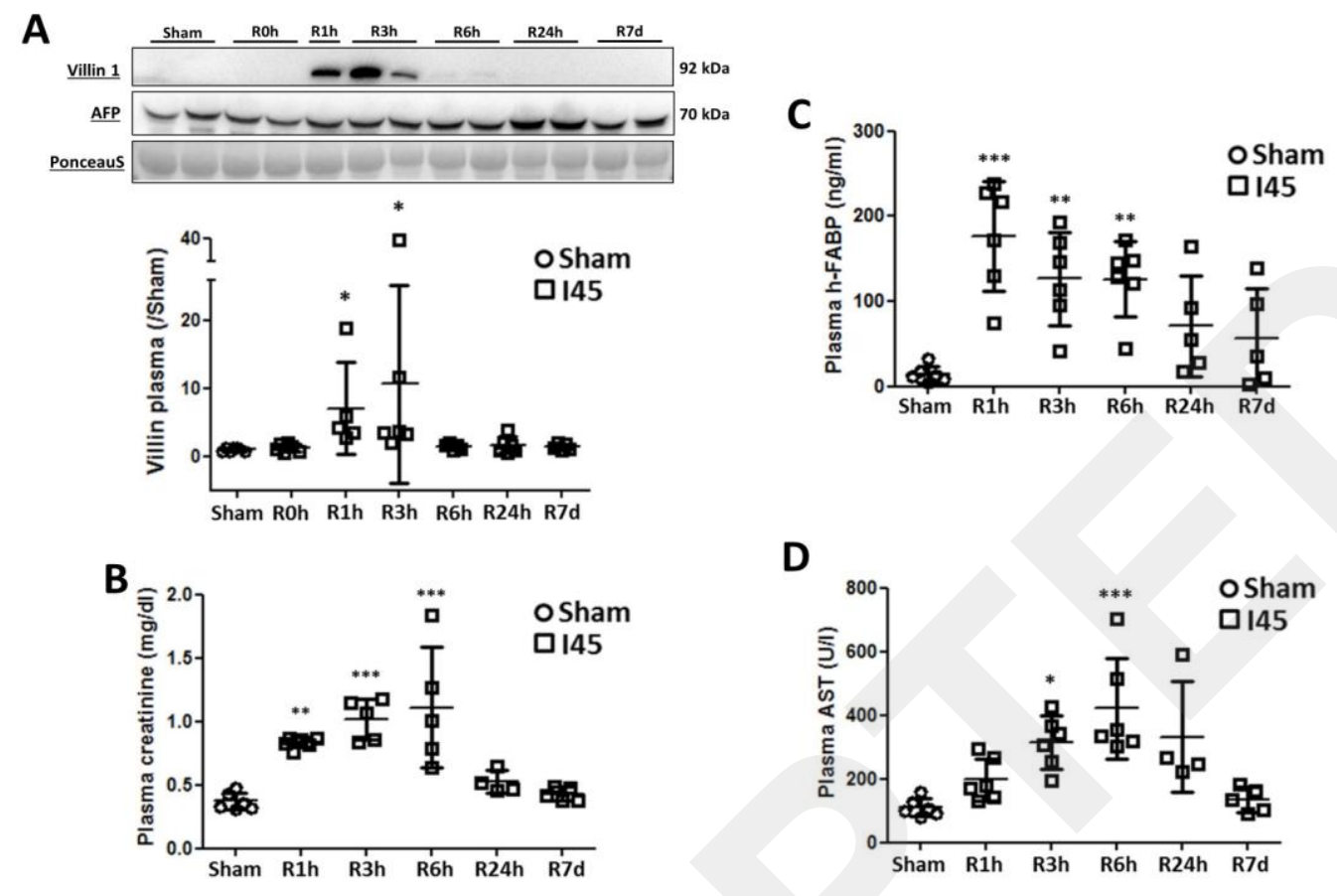

E
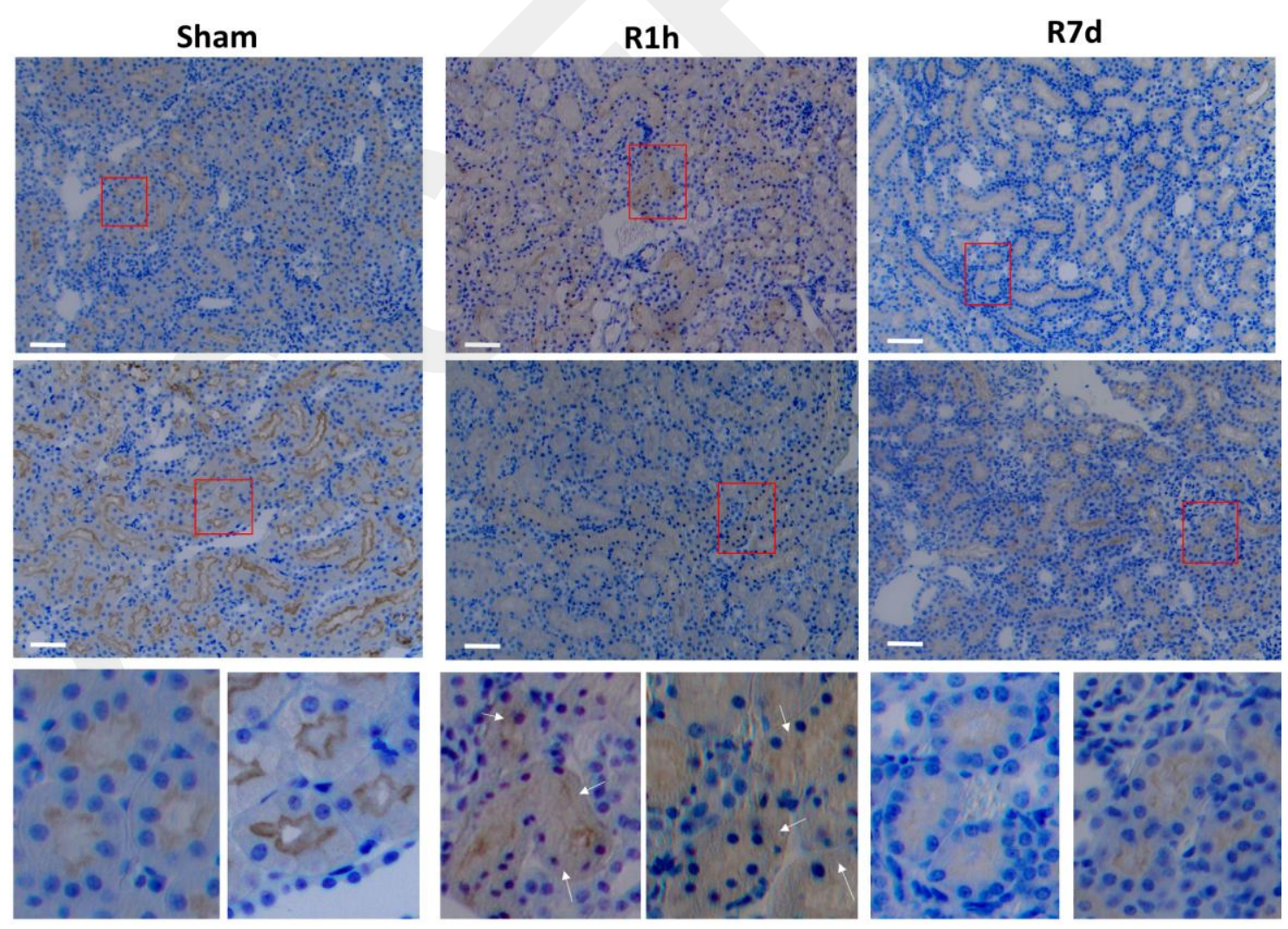
Figure 2
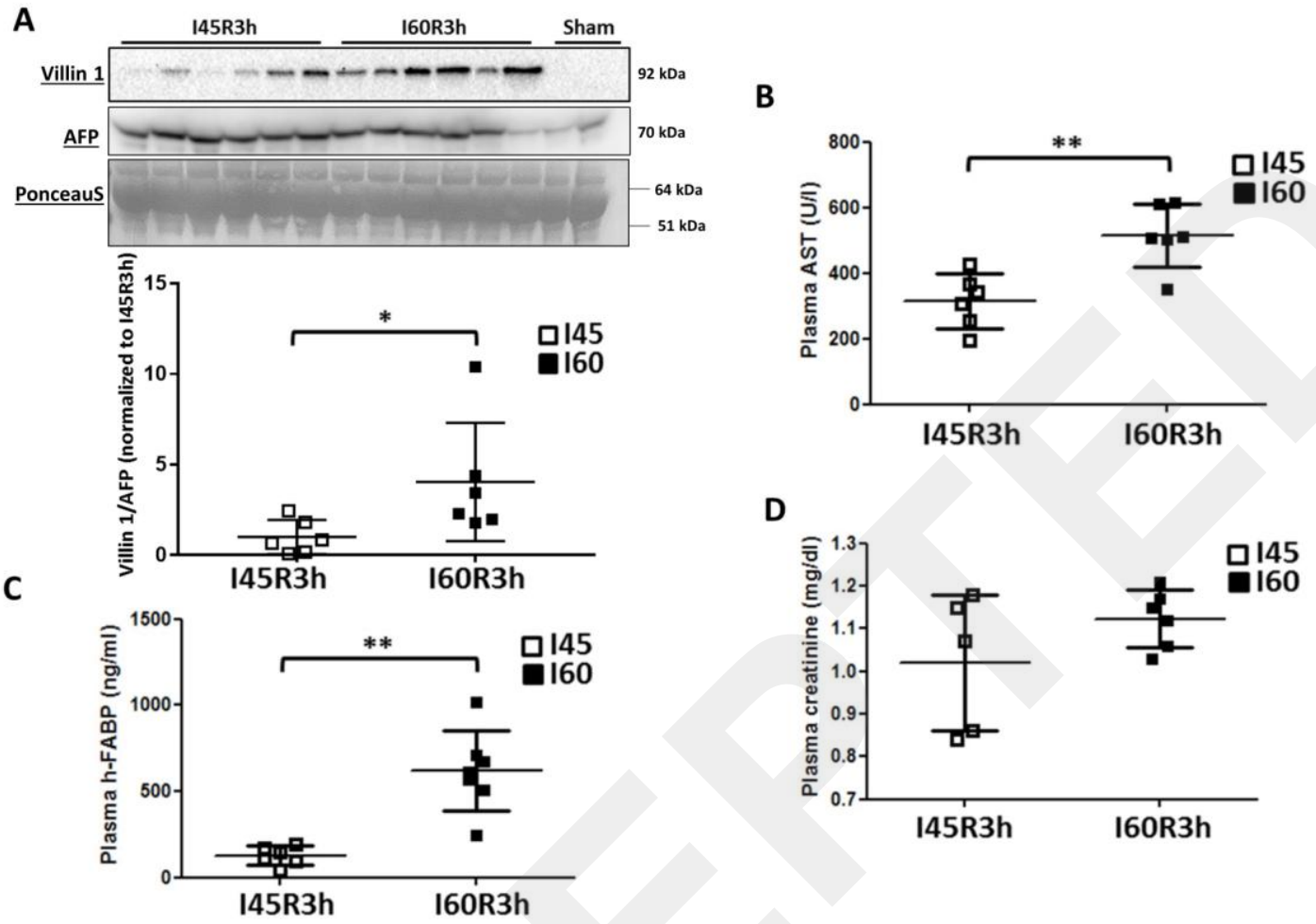
Figure 3

A

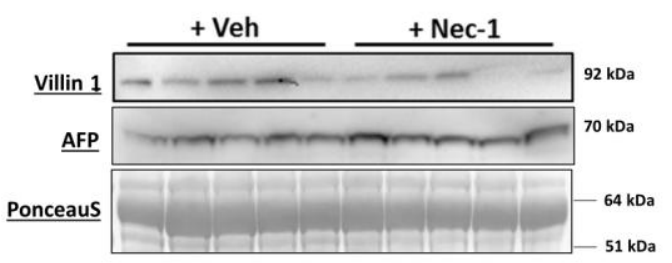

C


。

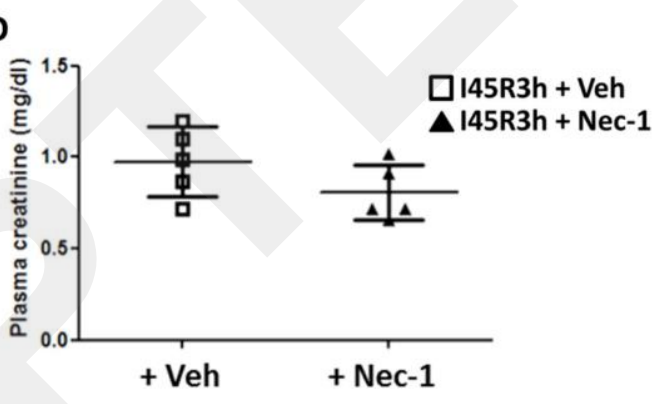

E

+ Veh

+ Nec-1
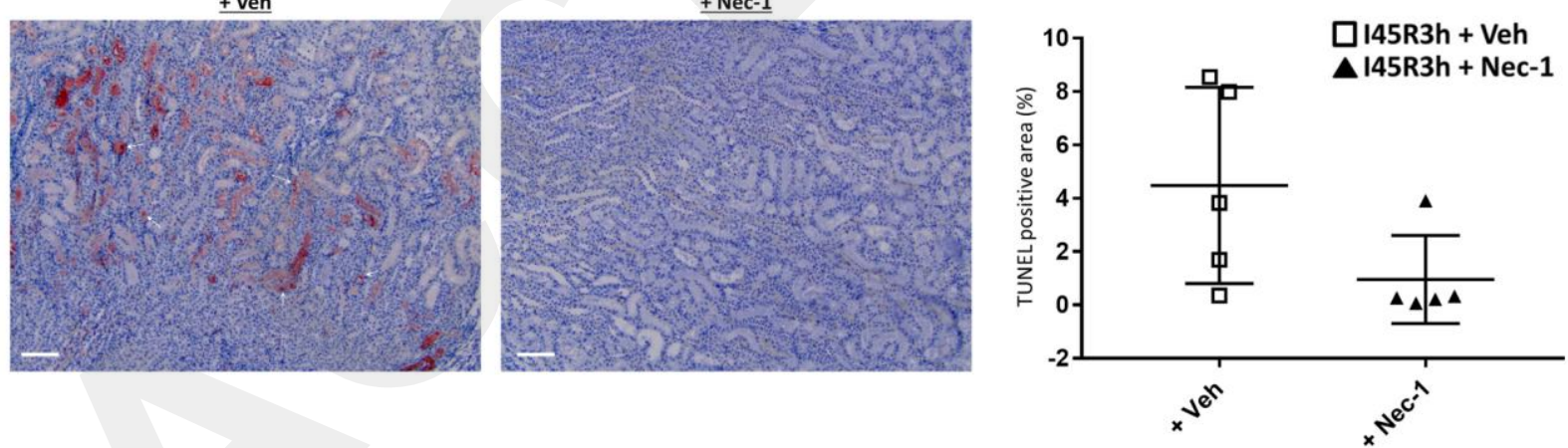
Figure 4

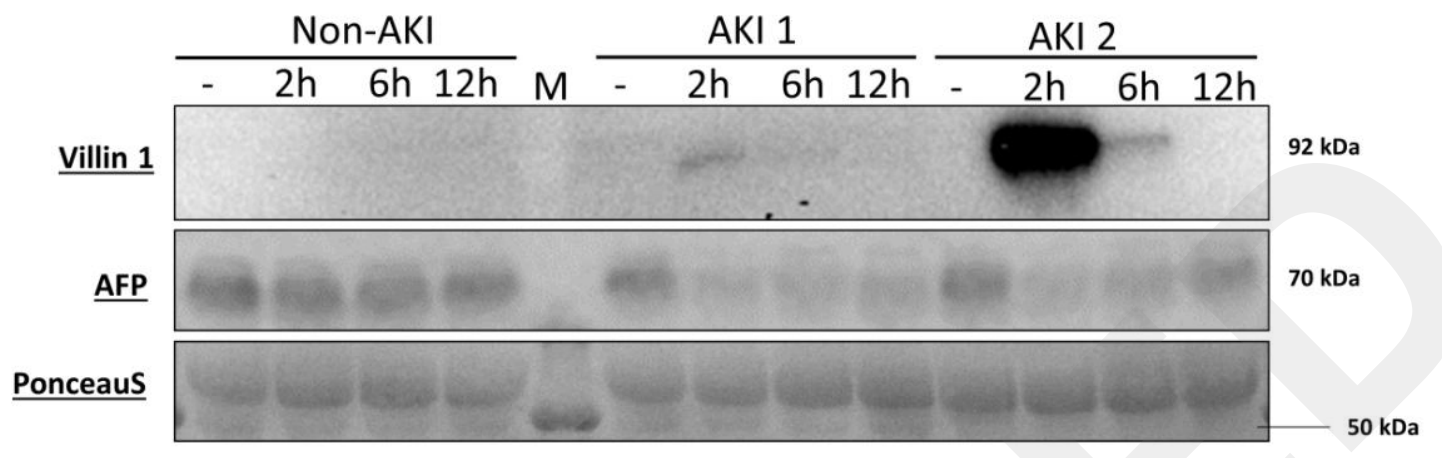




\section{Tables}

Table 1: overview of the donor and recipient characteristics and the other tested (plasmatic or urinary) biomarkers of liver transplantation patients that did (AKI) or did not (non-AKI) develop AKI

\begin{tabular}{|c|c|c|c|c|}
\hline & & $\begin{array}{l}\text { Patient } 1 \text { (non- } \\
\text { AKI) }\end{array}$ & Patient 2 (AKI) & Patient 3 (AKI) \\
\hline Recipient age (y) & & 58 & 57 & 61 \\
\hline Donor age (y) & & 75 & 76 & 56 \\
\hline Cold ischemia time (h:min) & & $6: 43$ & $11: 12$ & $7: 15$ \\
\hline Early allograft dysfunction & & no & yes & yes \\
\hline Peak AST (U/I) & & 675 & 6410 & 2486 \\
\hline \multirow[t]{4}{*}{ Creatinine (mg/dl) } & Baseline & 0.82 & 0.61 & 1.33 \\
\hline & $2 \mathrm{~h}$ & 0.81 & 0.79 & 1.45 \\
\hline & $6 \mathrm{~h}$ & 0.77 & 0.72 & 1.83 \\
\hline & $12 \mathrm{~h}$ & 0.79 & 0.72 & 2.37 \\
\hline \multirow[t]{4}{*}{ Plasma h-FABP baseline (ng/ml) } & Baseline & 4.40 & 14.85 & 7.36 \\
\hline & $2 \mathrm{~h}$ & 16.08 & 34.57 & 16.76 \\
\hline & $6 \mathrm{~h}$ & 20.43 & 56.20 & 39.20 \\
\hline & $12 \mathrm{~h}$ & 16.57 & 51.42 & 50.00 \\
\hline \multirow[t]{4}{*}{ Urine NGAL (ng/ml) } & Baseline & 29.55 & 0.20 & NA \\
\hline & $2 \mathrm{~h}$ & 22.45 & 0.20 & 6.38 \\
\hline & $6 \mathrm{~h}$ & 34.43 & 1.97 & 11.54 \\
\hline & $12 \mathrm{~h}$ & 53.27 & 2.86 & 4.60 \\
\hline \multirow[t]{4}{*}{ Urine KIM-1 (ng/ml) } & Baseline & 3.97 & 8.89 & NA \\
\hline & $2 \mathrm{~h}$ & 3.88 & 3.03 & 32.89 \\
\hline & $6 \mathrm{~h}$ & 5.90 & 4.77 & 53.73 \\
\hline & $12 \mathrm{~h}$ & 7.60 & 4.37 & 30.98 \\
\hline
\end{tabular}


Supplementary Figure 1: Plasmatic villin 1 detection in a porcine kidney autotransplantation model. The extent of graft injury is derived from the duration of warm ischemia the graft experienced: minimally injured ( $0 \mathrm{~min})$, moderately injured ( $30 \mathrm{~min}$ ) and severely injured (60 $\mathrm{min}$ ) before $18 \mathrm{~h}$ or cold storage. ${ }^{1}$ Plasma samples were collected 1 day before transplantation $(-24 \mathrm{~h})$, before reperfusion $(0 \mathrm{~h}), 15 \mathrm{~min}, 1 \mathrm{~h}, 3 \mathrm{~h}$ and $6 \mathrm{~h}$ postreperfusion and each following day (24 - 192 h). Plasma was tested for (A) villin 1 by Western blotting; (B) aspartate transaminase, AST; (C) heart-type fatty acid-binding protein, h-FABP and; (D) creatinine ( $N=6$ per group, $\mathrm{N}=7$ for severely injured grafts). Panels $\mathrm{A}-\mathrm{D}$ are reproduced with permission.

A
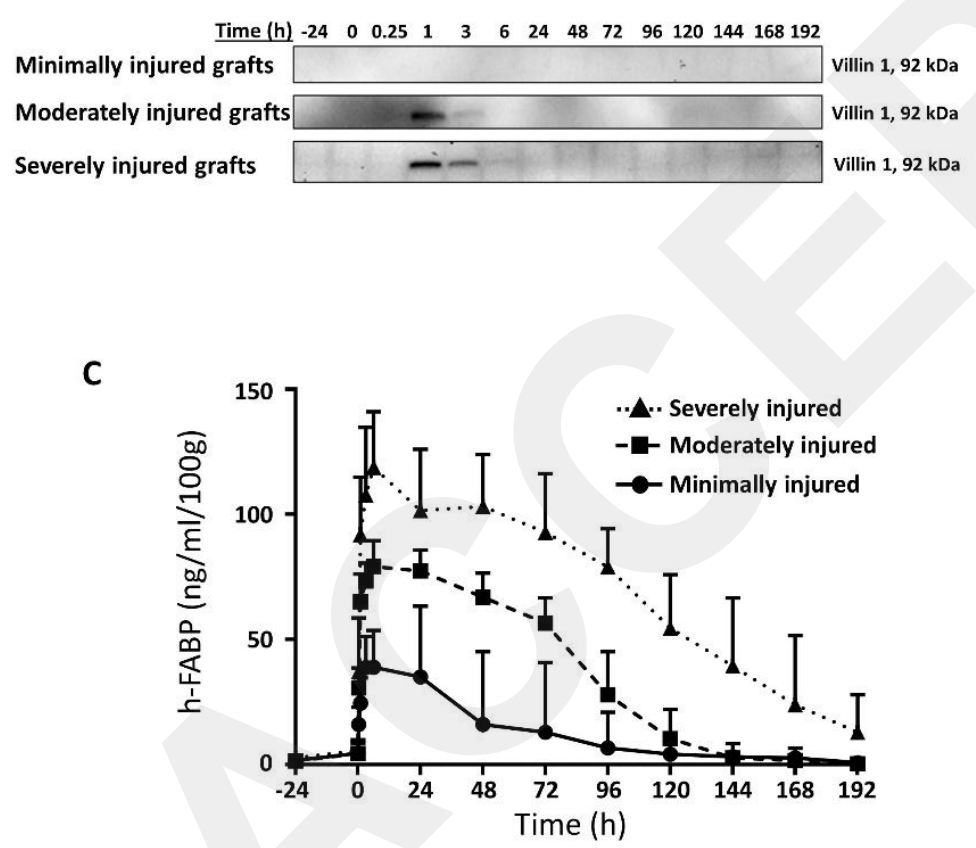
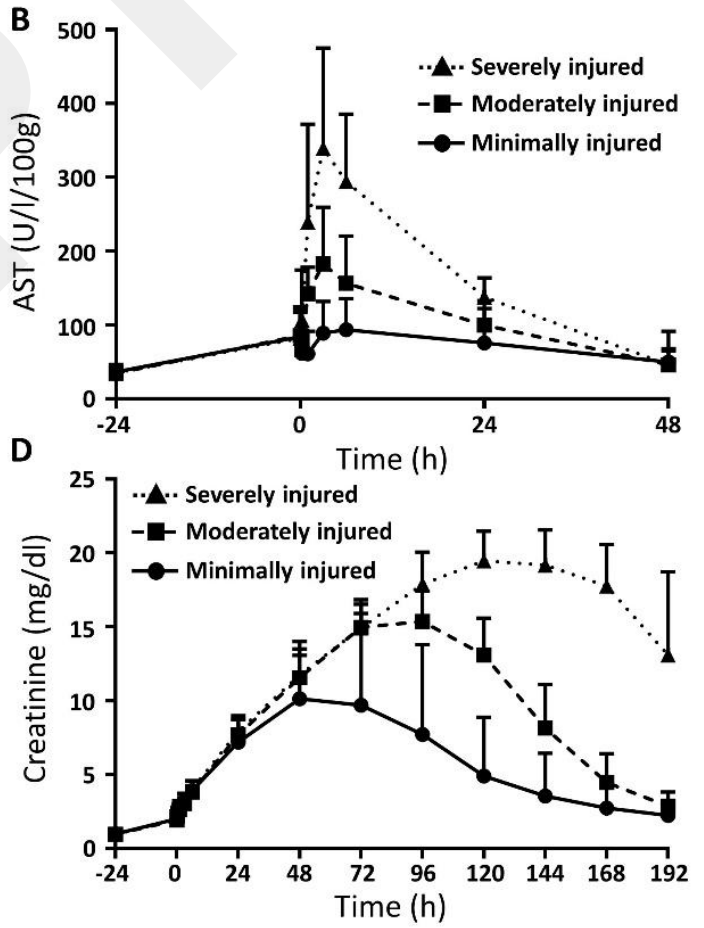

\section{References}

1. Jochmans I, Lerut E, van Pelt J, Monbaliu D, Pirenne J. Circulating AST, H-FABP, and

NGAL are early and accurate biomarkers of graft injury and dysfunction in a preclinical model of kidney transplantation. Ann Surg. 2011; 254(5):784-91. 